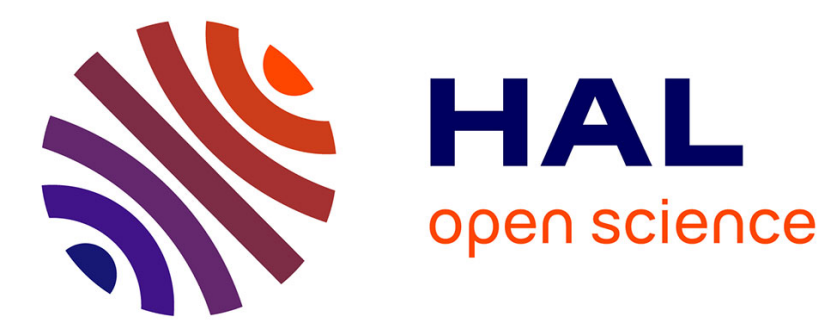

\title{
L'Europe et la laïcité contre la démocratie en Turquie
}

Jean-François Leguil-Bayart

\section{To cite this version:}

Jean-François Leguil-Bayart. L'Europe et la laïcité contre la démocratie en Turquie. Critique Internationale, 1998, 1, pp.15-22. 10.3406/criti.1998.1348 . hal-01010692

\section{HAL Id: hal-01010692 \\ https://hal-sciencespo.archives-ouvertes.fr/hal-01010692}

Submitted on 20 Jun 2014

HAL is a multi-disciplinary open access archive for the deposit and dissemination of scientific research documents, whether they are published or not. The documents may come from teaching and research institutions in France or abroad, or from public or private research centers.
L'archive ouverte pluridisciplinaire HAL, est destinée au dépôt et à la diffusion de documents scientifiques de niveau recherche, publiés ou non, émanant des établissements d'enseignement et de recherche français ou étrangers, des laboratoires publics ou privés.

\section{(1) $(1) \Theta$}

Distributed under a Creative Commons Attribution - NonCommercial - NoDerivatives| 4.0 


\section{L'Europe et}

la laïcité contre la démocratie en Turquie

par Jean-François Bayart

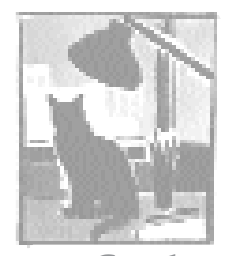

Contre-jour

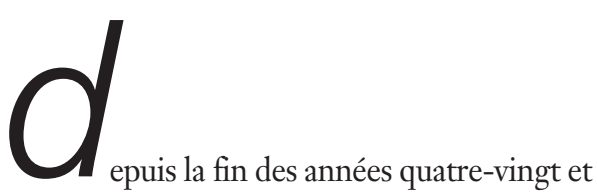

l'apogée du « règne » du président Turgut

Özal, une hypothèse dominait le débat politique en Turquie : celle de la consolidation de la démocratie, grâce à son intégration, sous une forme ou une autre, à l'espace européen et par le biais d'une réconciliation progressive entre les institutions néo-kémalistes et la problématique de l'islam politique. En deux ans, ce scénario a perdu beaucoup de sa vraisemblance. Non que les prémisses sur lesquelles il s'appuyait aient été infondées. Mais les événements, dans leur contingence, les ont fortement ébranlées. En 1997, le Refah - le parti islamique de Necmettin Erbakan - a été chassé du gouvernement sous la pression de l'armée, puis dissous. La mise en œuvre de l'Union douanière avec l'Union européenne (1995) a dérapé du fait de l'incapacité de Bruxelles à tenir ses engagements financiers à la suite du veto grec. Les conclusions du sommet de Luxembourg, en décembre dernier, ont provoqué un raidissement de la diplomatie turque qui était parfaitement prévisible, et qui cependant a semblé prendre au dépourvu les chancelleries européennes malgré l'intensité du dialogue politique qu'elles entretiennent avec Ankara. De façon inopinée, le 29 mai, la proposition de loi de l'Assemblée nationale française reconnaissant le génocide des Arméniens a ouvert une crise aiguë entre la Turquie et l'une de ses meilleures alliées au sein de l'UE. D'un incident à l'autre, les relations entre la Grèce, Chypre et la Turquie ont pris un tour particulièrement venimeux, tandis que les premiers pas d'un véritable partenariat militaire turco-israélien, inauguré par l'accord-cadre de février 1996, ont accru la tension avec la Syrie. Dans le même temps, les divisions de la classe politique ont empêché des réformes économiques indispensables, aggravant la crise financière.

Pour autant, le scénario « démo-européen » n'est pas devenu définitivement caduc, car il repose lui aussi sur des tendances lourdes. En outre on ne peut exclure un retournement de la conjoncture politique qui le favoriserait à nouveau, moyennant une réforme du mode de scrutin législatif, voire des institutions elles-mêmes, 
que réclame notamment le président Süleyman Demirel (non sans susciter de fortes résistances car il en serait le principal bénéficiaire). N'oublions pas que la décennie özalienne, qui a permis une libéralisation économique et politique sans précédent, faisait suite à une période particulièrement sombre de l'histoire du pays, marquée par la violence, le terrorisme et la répression militaire. Enfin, le sommet de Cardiff, en juin, a mis un peu de baume au cour de la diplomatie turque et, dans la foulée des initiatives de Tony Blair, la présidence autrichienne de l'UE se montre désireuse de faire progresser les choses.

Néanmoins, l'embellie est toute relative, et il importe de considérer aujourd'hui la vraisemblance d'un autre scénario, particulièrement préoccupant pour les intérêts européens : celui du free rider, qui verrait la Turquie maintenir l'essentiel de ses liens économiques avec l'UE, tout en développant une politique étrangère et de sécurité plus indépendante, au prix d'une crispation nationaliste dangereuse pour sa démocratie, pour sa stabilité interne et pour la sécurité régionale.

$\boldsymbol{L}$ a désinvolture avec laquelle les Quinze ont traité la Turquie à Luxembourg, et plus largement la légèreté avec laquelle l'Union européenne gère ce dossier, sous la pression grecque, depuis de longues années, paraissent constituer une grave erreur pour au moins cinq raisons :

1. Ce faisant, l'Europe renie sa propre signature, ou plutôt ses signatures, ce qui n'est pas de nature à accroître sa crédibilité politique sur le plan international : non seulement le Traité d'Ankara de 1963 - encore que les parties n'en aient pas la même lecture - mais aussi son engagement, souscrit lors de l'adhésion de la Grèce en 1981, à ne pas laisser interférer le contentieux gréco-turc dans ses relations avec la Turquie, ou encore sa promesse d'accompagner d'une aide financière la mise en œuvre de l'Union douanière de 1995.

2. L'Union européenne prend le risque de s'aliéner un État dont la position géostratégique est de première importance pour sa propre sécurité du fait de sa proximité avec la Russie, les Républiques caucasiennes et d'Asie centrale, la Grèce, les Balkans et le Moyen-Orient. La tension qui prévaut à Chypre et en mer Égée, ou le jeu mené par Ankara au Proche-Orient, ou encore le poids des choix effectués par la Turquie en faveur de l'opération « Tempête du désert » lors de la guerre du Koweit, mais qu'elle aurait fort bien pu ne pas faire compte tenu des réticences de son armée, démontrent que l'Union européenne est directement concernée par l'orientation de la politique étrangère de son alliée.

3. L'Union européenne s'interdit également d'avoir à l'égard de la Turquie une approche à la hauteur du potentiel économique de celle-ci, seul à même de donner au Partenariat euro-méditerranéen une substance autre que sécuritaire. Dans le monde d'aujourd'hui il paraît assez inconséquent de snober un pays qui affiche une croissance de 7 à $8 \%$ - quels que soient les déséquilibres structurels sur lesquels 
celle-ci repose - et qui offre des opportunités géographiques tangibles en matière de délocalisation industrielle.

4. L'Union européenne repousse non pas seulement la Turquie, mais également Istanbul qui se pense de plus en plus comme une ville « globale », qui représente la conurbation la plus importante du Bassin méditerranéen et un pôle industriel non négligeable, et qui a retrouvé, à la faveur de l'ouverture du bloc ex-soviétique et de la fermeture progressive de la forteresse Europe, son rôle de plaque tournante régionale en direction de l'espace russe, des Balkans, de l'Asie centrale, de l'Afrique du Nord, voire de l'Afrique subsaharienne.

5. Enfin, l'Union européenne, en excluant de facto toute perspective d'adhésion de la Turquie, reste un « club chrétien » et s'enferme, fût-ce à son corps défendant ou de façon honteuse, dans une conception culturaliste de sa citoyenneté. Celleci pose problème d'un point de vue philosophique, ne restera pas sans conséquences politiques sur l'intégration de ses propres citoyens de confession musulmane, affaiblit sa capacité de rayonnement dans le monde arabo-musulman et, de façon générale, sa vocation à devenir un acteur majeur du processus de globalisation.

Cette fantastique erreur de jugement qui culmine avec le sommet de Luxembourg s'explique en partie par des facteurs politiques immédiats, tels que la rigidité de la diplomatie grecque et les préoccupations électoralistes du chancelier Kohl à quelques mois d'une consultation délicate pour lui. Mais elle témoigne plus profondément d'une double incompréhension des Européens à l'endroit de la Turquie.

Incompréhension, d'abord, sans doute feinte, de ce que demande réellement, au moins dans l'immédiat, Ankara : non l'adhésion, mais la reconnaissance que celleci n'est pas exclue ou repoussée aux « calendes grecques », au sens littéral de l'expression. Bien sûr, les chancelleries ouest-européennes, ou tout au moins certaines d'entre elles, admettent désormais la « vocation européenne » de la Turquie. Mais, Conférence européenne ou pas, la décision de Luxembourg a vidé cette pétition de principe de l'essentiel de sa substance dès lors que la Turquie n'apparaît pas en bonne et due forme sur la liste des éligibles. Et ce, même si une lecture attentive du communiqué des Quinze laisse réellement ouverte la porte à la candidature turque, comme se plaisent à le souligner leurs diplomates. Ankara a beau jeu de faire valoir que les Européens exigent en fait de la Turquie le respect préalable des critères dits de Copenhague, alors qu'ils en attendent l'application par les autres candidats à l'issue du processus de négociation. Il eût mieux valu entériner l'éligibilité théorique de la Turquie et arguer des obstacles indéniables, tant politiques que démographiques et économiques, qui s'opposent à l'ouverture des négociations dans un avenir prévisible. La discrimination dont Ankara a été l'objet à Luxembourg a été gratuite et inévitablement humiliante pour un pays qui a le nationalisme à fleur de peau. 
Incompréhension, ensuite, de l'historicité européenne de la Turquie, quoi qu'en pense un Samuel Huntington. L'Empire ottoman a été le légataire universel de Byzance dont il a perpétué les formes d'organisation politique, en particulier sur le plan des relations entre l'État et le religieux, et maintes pratiques culturelles, par exemple dans les domaines de la musique ou de la cuisine. L'islamisation quasi totale de l'Anatolie est un phénomène récent, heureusement irréductible à ses formes les plus tragiques telles que l'échange des populations, les conversions forcées ou les massacres. Elle est de toute façon indissociable, même de ce point de vue, d'un fait moderne ancré dans l'histoire européenne, celui du nationalisme et de la généralisation de l'État-nation comme forme de souveraineté politique. Or, précisément, le nationalisme turc participe directement de la matrice historique du nationalisme européen. Soit que ses théoriciens, originaires des provinces balkaniques ou des États et provinces turcophones de l'espace russe, aient découvert cet imaginaire politique de pair avec les nationalistes d'Europe centrale et orientale. Soit qu'ils aient baigné dans l'atmosphère positiviste, comtienne ou durkheimienne, mais également nationaliste, de l'Université française. L'Empire ottoman, puis la Turquie kémaliste n'ont cessé d'être parties prenantes au champ intellectuel, idéologique et politique de l'Europe. Le rôle de Bartok dans la codification et la standardisation de la musique populaire anatolienne entre les deux guerres - conformément au processus d'invention de la modernité par « invention de la tradition », identifié par Eric Hobsbawm et Terence Ranger - est plus qu'une curiosité. Il symbolise le lien organique qui unit la trajectoire turque au destin de l'ensemble du continent. Pareillement, les mobilisations politico-identitaires les plus contemporaines, à savoir l'islam politique et la revendication nationaliste kurde, procèdent en grande partie de l'émigration turque dans la « banane » urbaine et industrielle de l'Europe occidentale.

Se refuser à considérer l'« européanéité » de la Turquie, c'est se priver, pour l'UE, d'une formidable réserve de puissance et de croissance, quels que soient les obstacles, non moins formidables, qui rendent pour le moment inenvisageable la réalisation de ce « capital ». C'est borner arbitrairement, et de façon malthusienne, la « frontière » du Vieux Continent, dans un contexte de nouvelle régionalisation du système international.

Quoi qu'il en soit, l'Union européenne, qui avait su (ou pu), par son élargissement, rendre irréversible la transition politique en Grèce, en Espagne et au Portugal, a renoncé, avec la décision de Luxembourg, à peser sur le cours de la démocratie en Turquie. Plus grave encore, elle a ruiné le fonds de commerce des partis démocratiques (ou en tout cas traditionnels) qui, sous divers avatars, ont assuré depuis le milieu du siècle la stabilité relative du parlementarisme et opposé aux interventions répétées de l'armée leur résilience surprenante. Depuis décembre, cette classe politique, qui n'avait pas besoin de cela pour connaître le discrédit, n'a plus 
de projet. La problématique nationaliste, qui enregistrait depuis le début des années quatre-vingt-dix une montée électorale régulière, même si le MHP n'en était pas toujours le bénéficiaire, s'en est trouvée soudain relégitimée de façon spectaculaire. Évolution d'autant plus inquiétante que l'affaire de Susurluk ${ }^{1}$ a mis en pleine lumière la véritable osmose entre la droite ultra-nationaliste ou nationaliste, le terrorisme d'État, les milieux criminels et l'économie politique de la guerre civile dans l'Est.

Simultanément, l'armée, sous couvert de défense de la laïcité, mais aussi sans doute pour promouvoir ses intérêts économiques contre la montée d'une nouvelle catégorie d'opérateurs liés au Refah, a remis sous pression le système politique. Première manifestation du délitement de la conditionnalité démocratique, l'interdiction du parti islamique a été facilitée par la suspension du dialogue politique entre Ankara et l'Union européenne, au lendemain du sommet de Luxembourg. Elle laisse entière la question de la représentation politique d'un cinquième de l'électorat et ne fait pas cas de la double contribution du Refah à la consolidation de la démocratie en Turquie : l'intégration aux institutions de la République parlementaire de l'exode rural et du particularisme kurde. Bien entendu cette fonction tribunitienne qu'assumait la formation de Necmettin Erbakan, un peu à l'image de ce qu'avaient fait en Europe occidentale les partis communistes et démocrates-chrétiens, ou en Pologne l'Église catholique, peut être reprise par son légataire, le Parti de la Vertu (Fazilet Partisi, FP). Reste à savoir si l'armée l'acceptera : la condamnation à dix mois de prison du maire d'Istanbul, Recep Tayyip Erdogan, le successeur le plus crédible d'Erbakan, et le resserrement de l'étau autour de Fetullah Gülen - le leader des Fetullahçi, un puissant mouvement néo-confrérique, moderniste et pro-européen - donnent aujourd'hui à penser que l'État-Major ne l'entend pas de cette oreille et continue de raisonner dans les termes d'un jeu à somme nulle entre l'islam et le kémalisme, même si le porte-drapeau des « faucons », le général Bir, a perdu du terrain à l'occasion du mouvement de nominations dans la haute hiérarchie militaire, début août.

Or, historiquement, la République s'est constituée moins contre l'islam que sur le mode de son contrôle, dans la continuité de l'Empire ottoman. Elle a poursuivi, en la modernisant, la bureaucratisation du champ religieux que celui-ci avait garantie au fil des siècles. De ce fait, l'islam s'est à son tour « kémalisé », adoptant notamment l'idéologie positiviste. La contradiction entre les deux univers mentaux et moraux est donc loin d'être évidente. C'est justement cette interaction qui est sous-jacente au parlementarisme des partis successifs que Necmettin Erbakan a dirigés et aux nombreux compromis que ce dernier a noués avec les militaires ou les autres formations politiques, de la fin des années soixante jusqu'au lendemain de l'interdiction du Refah. 
De même, sociologiquement, les choses ne se présentent pas comme une simple alternative entre l'islam et la laïcité. En juin, par exemple, Mme Nazli Ilicak, membre en vue du Comité directeur du Parti de la Vertu, a défrayé la chronique en servant de l'alcool lors de la réception qu'elle offrait en l'honneur de son président, Recai Kutan. En réponse aux attaques dont elle a été alors l'objet, elle a rétorqué que, de l'alcool, elle en buvait elle-même et qu'elle ne changerait pas de comportement : « À ma connaissance, le Parti de la Vertu n'est pas une confrérie, mais un parti politique qui s'adresse à tout le monde en leur demandant un soutien électoral. » « C'est une femme qui en a », a concédé $M$. Kutan, dans le style macho qui sied à un leader turc, et son porte-parole a précisé que «le FP ne s'occupe pas du mode de vie des gens, mais de la politique nationale ». Quant au quotidien islamique radical Akit, il avait à s'indigner, dans son édition du 24 octobre 1997, du comportement des jeunes filles voilées : « On les voit chanter, danser, se déhancher et se pâmer d'aise dans les concerts de pop music turque, essayant d'approcher les chanteurs masculins pour les enlacer ou les embrasser. C'est en totale contradiction avec la morale musulmane et le foulard qu'elles portent. Il est temps de se poser sérieusement la question : où allons-nous ? » Interrogation cruciale, en effet, si l'armée devait persister à contraindre les acteurs politiques et sociaux à choisir entre des identifications qui sont en réalité mêlées et à les acculer en quelque sorte à renoncer à une part d'eux-mêmes.

La même observation peut être faite au sujet de la revendication kurde. Il devient chaque jour plus clair que le PKK ne demande pas l'indépendance, ni même peut-être une autonomie territoriale. Lidentité kurde doit désormais beaucoup à la diaspora vivant en Europe de l'Ouest. Comme le montre bien Martin van Bruinessen, son rapport au Kurdistan est maintenant d'ordre fantasmatique, comparable, mutatis mutandis, à la relation des sionistes ou des Irlandais américains à Israël ou à l'Eire. Mais, si l'armée ne peut rien contre les images de Med TV, diffusées par satellite à partir de Londres, elle peut continuer à ratisser les montagnes et à entretenir un conflit qui représente une dépense - et donc, pour certains, une ressource - de 8 milliards de dollars par an, sans compter les revenus narcotiques ou contrebandiers qu'il permet de dégager. Néanmoins, le vrai coût (ou le vrai profit) de la guerre est d'ordre politique. Ses bénéficiaires sont les secteurs ultra-nationalistes, souvent liés à l'économie internationale du crime, dont nous savons qu'ils représentent la principale menace pour la démocratie turque, et aussi peut-être, paradoxalement, pour son unité. En effet la problématique identitaire qu'ils incarnent est en porte-à-faux avec l'hétérogénéité humaine du pays, occultée par l'idéologie kémaliste, mais qui ressurgit à la faveur de la chute de l'Empire soviétique, des guerres du Caucase, de l'explosion de la Yougoslavie et de la « globalisation ». La stratégie de la tension, au sens italien du terme, que les milieux ultra-nationalistes mènent contre la communauté alevi, en particulier, conforte la 
« communautarisation » de cette dernière. Une tendance que favorisent de toute façon, entre autres facteurs, les conditions sociales de l'exode rural et d'une urbanisation très rapide, par quartiers d'« originaires », et à laquelle le PKK et le mouvement d'extrême-gauche Dev-Sol proposent aujourd'hui une expression armée. À forcer les gens à choisir entre des répertoires identitaires qu'ils combinent spontanément, on prend le risque de les voir ne pas privilégier « le bon ». Si la «balkanisation » de la Turquie est peu plausible dans l'état actuel des choses, une résurgence de la violence des années soixante-dix n'est pas à exclure. Elle légitimerait aux yeux de l'armée et de l'étranger un retour à l'autoritarisme dont la crispation nationaliste serait l'inévitable contrepartie idéologique.

Répétons-le : une telle évolution ne remettrait pas en cause les liens économiques avec l'Union européenne, qui semblent désormais irréversibles². Mais elle pourrait amener la Turquie à en renégocier les bases au vu de ses déconvenues récentes et surtout la pousser à une politique étrangère et de sécurité beaucoup plus autonome, sans nécessairement qu'elle reconsidère son appartenance à l'Alliance atlantique, ni à l'inverse qu'elle développe une relation stratégique exclusive avec les ÉtatsUnis et Israël en substitution de son ancrage européen. Cependant, les nuisances d'une telle stratégie pourraient s'avérer considérables pour l'Europe en introduisant une marge supplémentaire d'imprévisibilité dans un jeu régional déjà très instable, et que complexifiera encore l'exploitation du pétrole de la Caspienne. Le spectre d'une guerre entre un pays de l'Union européenne et un adversaire extérieur deviendrait palpable avec la dramatisation du contentieux gréco-turc et l'affaiblissement de l'intermédiation européenne. Enfin, ce cours nationaliste pourrait se traduire à la longue par la volonté de se doter de l'arme nucléaire, que justifieraient l'entrée dans le club de l'Inde, du Pakistan, voire à terme de l'Iran et la nécessité de garantir l'indépendance nationale dans un contexte de crise de confiance généralisée entre l'Europe et Ankara.

Depuis la Seconde Guerre mondiale, la Turquie a connu une succession de cycles d'ouverture et de fermeture sur les plans économique et politique, mouvement de systole et de diastole qui s'est longtemps énoncé à travers les thématiques de la laïcité, de la démocratie, de la société civile, de l'économie de marché et de la libéralisation. On peut en tirer argument pour relativiser la portée des derniers événements. Mais cette évolution du pays reposait sur l'évidence de son ancrage à l'Ouest, que dictait la guerre froide, et sur la perspective européenne.

Ces deux facteurs se sont évanouis, accroissant la marge d'incertitude. Dans les années quatre-vingt, la société turque a amorcé une vraie mutation. Eu égard aux dichotomies classiques et même stéréotypiques - « La Turquie à la croisée des chemins », « La Turquie entre l'Est et l'Ouest », « islam ou laïcité »- l'özalisme a en définitive permis l'émergence de ce que l'on pourrait nommer, toujours par 
référence à l'Italie, une « Troisième Turquie », grâce à la libéralisation économique et à l'acceptation tacite d'une recomposition entre la problématique républicaine et celle de l'islam. La comparaison avec l'Italie vaut d'ailleurs également par la montée en puissance, à la faveur de ce processus, de nouveaux pôles industriels particulièrement dynamiques en Anatolie - par exemple à Denizli, à Konya, à Mersin ou à Gaziantep - et d'un patronat économiquement très entreprenant et politiquement conservateur, souvent proche, précisément, du Refah ou des courants islamiques au sein des partis de la droite traditionnelle. On peut aussi envisager, dans cette situation, une sorte de movida qui verrait une transformation notable des mœurs urbaines, en particulier de la jeunesse, parfois sous le couvert de pratiques islamiques, et dont certains signes sont déjà perceptibles... au grand dam de l'éditorialiste d'Akit !

La conséquence la plus grave du raidissement laïciste de l'armée et de l'impasse turco-européenne est de contrarier l'affirmation de cette « Troisième Turquie », qui seule pourrait dépasser les fausses alternatives identitaires et les polarisations politiques qu'elles engendrent.

\footnotetext{
1. Le 3 novembre 1996, l'accident d'une voiture dans la bourgade de Susurluk confirma les assertions de plusieurs députés et de l'hebdomadaire Aydinlik sur les responsabilités de Mehmet Agar, le ministre DYP de l'Intérieur et précédemment chef de la police, dans le «triangle police-mafia-politique », dont l'existence avait déjà été démontrée quelques mois auparavant par les scandales du « gang des frères Söylemez » dans la région d'Urfa (juin) et du « gang en uniforme »à Hakkari (septembre). Se trouvaient à bord du véhicule, outre la maîtresse de l'un des passagers : Sedat Bucak, député DYP d'Urfa et leader d'une milice de plusieurs centaines de supplétifs de l'armée ; Husseyin Kocadag, un haut fonctionnaire alevi de la police qui était l'un des fondateurs des Özel Tim spécialisés dans la lutte contre le PKK et qui avait joué un rôle de médiation lors de l'émeute confessionnelle du quartier de Gazi, à Istanbul, en mars 1995, plus ou moins attisée par les gauchistes de Dev-Sol ; et enfin Abdullah Catli, militant ultra-nationaliste compromis dans l'attentat contre Jean-Paul II, recherché par Interpol après son évasion d'une prison suisse où il était incarcéré pour trafic de stupéfiants, et détenteur d'une fausse carte de police au moment des faits. Malgré l'ampleur de la mobilisation de l'opinion publique, cette affaire de Susurluk est loin d'avoir été éclaircie. Et le commando qui a tenté d'assassiner Akin Birdal, le président de l'Association turque des droits de l'homme, le 12 mai dernier, s'est avéré avoir été entrainé par un sous-officier de la Gendarmerie, dans une caserne des environs d'Istanbul, et faire lui aussi partie du « gang de Susurluk »...

2. Les transactions avec l'UE représentent environ la moitié du total du commerce extérieur turc : 46,6\% des exportations, $51 \%$ des importations en 1997. Le commerce bilatéral Turquie/UE est de plus en plus déficitaire pour la Turquie. Mais le rythme d'augmentation de ce déficit - inévitable du fait de l'entrée en vigueur de l'Union douanière en 1996, et prévu s'est ralenti en 1997. En outre, les biens intermédiaires et les biens d'équipement, indispensables à l'investissement, ont représenté l'essentiel des importations turques, les biens de consommation n'entrant que pour 10\% dans celles-ci. De façon générale, l'intégration de l'économie turque à l'économie de l'UE est la condition nécessaire à sa modernisation et à l'amélioration de sa position dans la division internationale du travail. Il est révélateur que l'association des hommes d'affaires proches de la mouvance islamique, la MÜSIAD, ne cache pas son intérêt pour le marché européen, nonobstant les prises de position politiques anti-européennes de Necmettin Erbakan.
} 